\title{
Holographic study of Wilson loop in the anisotropic back- ground with confinement/deconfinement phase transition
}

\author{
Pavel Slepov ${ }^{1 *}$ \\ ${ }^{1}$ Moscow State University, Faculty of Physics, 1-2 Leninskie Gory, 119991, Moscow, Russia
}

\begin{abstract}
Within the bottom-up holographic QCD using anisotropic black brane solutions in 5D Einstein-dilaton-two-Maxwell system constructed in $[1,2]$, we study the temporal Wilson loops with arbitrary orientation in respect to the anisotropy direction. We calculate the minimal surfaces of the corresponding probing open string world-sheet in anisotropic backgrounds with various temperatures and chemical potentials. The dynamical wall locations, providing the quark confinement, depend on the orientation of the quark pairs, that gives a crossover transition line between confinement/deconfinement phases in the dual gauge theory.
\end{abstract}

\section{Introduction}

Study of the QCD phase diagram, as a function of temperature $T$ and chemical potential $\mu$, is one of the most important problems. The AdS/CFT duality provides an alternative tool for dynamics understanding of quark-gluon plasma (QGP) produced in the heavy-ions-collisions (HIC) [3-6].

It is believed that there are indications that QGP is anisotropic right after the collision of ions. Isotropisation occures at times $\sim 2 \mathrm{fm} / \mathrm{s}$. It is natural to consider an anisotropic metric within the holographic approach. Anisotropy is usually provided by adding magnetic ansatz of Maxwell field to dilaton gravity action. Non-zero chemical potential is introduced via electic ansatz for the second Maxwell field. Thereby the 5-dimensional dilaton gravity with two Maxwell fields turns out to be the most suitable model. Such model was considered in $[1,7]$. In the simplest case anisotropic Lifshitz-like models, characterized by anisotropic parameter $v$, have been investigated [8].

We consider a 5-dimensional Einstein-dilaton-two-Maxwell system. In the Einstein frame the action of the system is specified as

$$
S=\int \frac{d^{5} x}{16 \pi G_{5}} \sqrt{-\operatorname{det}\left(g_{\mu \nu}\right)}\left[R-\frac{f_{1}(\phi)}{4} F_{(1)}^{2}-\frac{f_{2}(\phi)}{4} F_{(2)}^{2}-\frac{1}{2} \partial_{\mu} \phi \partial^{\mu} \phi-V(\phi)\right],
$$

where $F_{(1)}^{2}$ and $F_{(2)}^{2}$ are the squares of the Maxwell fields $F_{\mu \nu}^{(1)}=\partial_{\mu} A_{\nu}-\partial_{v} A_{\mu}$ and $F_{\mu \nu}^{(2)}=$ $q d y^{1} \wedge d y^{2}, f_{1}(\phi)$ and $f_{2}(\phi)$ are the gauge kinetic functions associated with the corresponding Maxwell fields, $V(\phi)$ is the potential of the scalar field $\phi$.

\footnotetext{
*e-mail: slepov@mi.ras.ru
} 
To find the black brane solution in the anisotropic background, we used the metric ansatz in the following form:

$$
\begin{aligned}
& d s^{2}=G_{\mu \nu} d x^{\mu} d x^{\nu}=\frac{L^{2} b(z)}{z^{2}}\left[-g(z) d t^{2}+d x^{2}+z^{2-\frac{2}{\nu}}\left(d y_{1}^{2}+d y_{2}^{2}\right)+\frac{d z^{2}}{g(z)}\right], \\
& \phi=\phi(z), \quad A_{\mu}^{(1)}=A_{t}(z) \delta_{\mu}^{0}, \\
& F_{\mu \nu}^{(2)}=q d y^{1} \wedge d y^{2},
\end{aligned}
$$

where $b(z)$ is the warp factor and $g(z)$ is the blackening function; we set the $\operatorname{AdS}$ radius $L=1$. All the quantities in formulas and figures are presented in dimensionless units.

Note that in $[1,2]$ the following strategy to study holographic model is used. Firstly, one takes $b$ suitable for phenomenological application, in particular one can take $b=e^{\frac{c z^{2}}{2}}$. Secondly, the anisotropic multiplier $z^{2-2 / v}$ is also fixed by phenomenological reasons [8]. Thirdly, one takes a specific function $f_{1}$ by reasons of simplicity. And finally, using E.O.M. following from (2), one finds coupling function $f_{2}$, potential $V$, Maxwell field potential $A_{\mu}$ and blackening function $g$. The last one has the form:

$$
g=1-\frac{z^{2+\frac{2}{v}}}{z_{h}^{2+\frac{2}{v}}} \frac{\mathfrak{G}\left(\frac{3}{4} c z^{2}\right)}{\mathfrak{5}\left(\frac{3}{4} c z_{h}^{2}\right)}-\frac{\mu^{2} c z^{2+\frac{2}{v}} e^{\frac{c z_{h}^{2}}{2}}}{4\left(1-e^{\frac{c c_{h}^{2}}{4}}\right)^{2}}\left(\mathfrak{b}\left(c z^{2}\right)+\frac{\mu^{2} c z^{2+\frac{2}{v}} e^{\frac{c z_{h}^{2}}{2}}}{4\left(1-e^{\frac{c z_{h}^{2}}{4}}\right)^{2}} \frac{\mathfrak{5}\left(\frac{3}{4} c z^{2}\right)}{\mathfrak{5}\left(\frac{3}{4} c z_{h}^{2}\right)}\left(\mathfrak{5}\left(c z_{h}^{2}\right),\right.\right.
$$

where the function $6(x)$ has the following expansion:

$$
\mathfrak{5}(x)=\sum_{n=0}^{\infty} \frac{(-1)^{n} x^{n}}{n !\left(1+n+\frac{1}{v}\right)}
$$

\section{The Wilson loop}

The purpose of our consideration is to calculate the expectation value of the temporal Wilson loop

$$
W\left[C_{\vartheta}\right]=e^{-S_{\vartheta, t}},
$$

oriented along vector $\vec{n}$, such that

$$
n_{x}=\cos \vartheta, \quad n_{y}=\sin \vartheta
$$

Following the holographic approach [9-11] we have to calculate the value of the Nambu-Goto action for test string in our background:

$$
S=-\frac{1}{2 \pi \alpha^{\prime}} \int d \xi^{0} d \xi^{1} \sqrt{-\operatorname{det} h_{\alpha \beta}},
$$

where

$$
h_{\alpha \beta}=G_{\mu \nu} \partial_{\alpha} X^{\mu} \partial_{\beta} X^{\nu}
$$

and $G_{\mu \nu}$ is given by (2). The world sheet presented in Fig. 1 is parameterized as

$$
X^{0} \equiv t=\xi^{0}, X^{1} \equiv x=\xi^{1} \cos \vartheta, X^{2} \equiv y_{1}=\xi^{1} \sin \vartheta, X^{3} \equiv y_{2}=\text { const }, X^{4} \equiv z=z\left(\xi^{1}\right)
$$




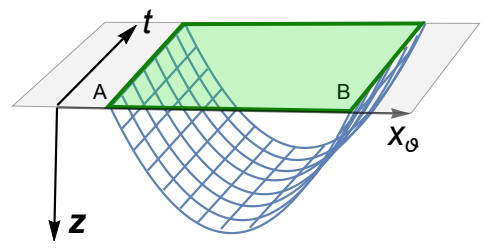

A

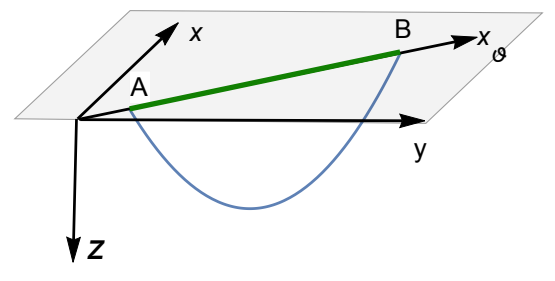

B

Figure 1. A) Wilson loop and the world sheet. B) Projection of the world sheet to fixed $t$

We can use the following notation $\xi \equiv \xi^{1}$. In our case the action can be rewritten:

$$
S=-\frac{\tau}{2 \pi \alpha^{\prime}} \int d \xi M(z(\xi)) \sqrt{\mathcal{F}(z(\xi))+\left(z^{\prime}(\xi)\right)^{2}},
$$

where $\tau=\int d \xi^{0}$,

$$
\begin{gathered}
M(z(\xi))=\frac{b(z(\xi))}{z(\xi)^{2}}, \\
\mathcal{F}(z(\xi))=g(z(\xi))\left(z(\xi)^{2-\frac{2}{v}} \sin ^{2}(\theta)+\cos ^{2}(\theta)\right) .
\end{gathered}
$$

The right-hand side of (12) defines the action of 1-dim dynamical model. This system possesses the first integral of motion:

$$
I=\frac{M(z) \mathcal{F}(z)}{\sqrt{\mathcal{F}(z)+z^{\prime 2}}} .
$$

If we introduce $z_{\min }$ at which $z^{\prime}(\xi)=0$, then the first integral can be expressed as:

$$
M\left(z_{\text {min }}\right) \sqrt{\mathcal{F}\left(z_{\text {min }}\right)}=I,
$$

Let us introduce the effective potential:

$$
\mathcal{V}(z(\xi)) \equiv M(z(\xi)) \sqrt{\mathcal{F}(z(\xi))}
$$

In order to calculate the length of the curve, at first we have to find the character length of string:

$$
\ell=\int_{0}^{z_{\min }} \frac{2}{\sqrt{\mathcal{F}(z)}} \frac{d z}{\sqrt{\left(\frac{\mathcal{V}(z)}{\mathcal{V}\left(z_{\min }\right)}\right)^{2}-1}}
$$

where $\mathcal{V}(z) \equiv M(z) \sqrt{\mathcal{F}(z)}$.

\section{Confinement/deconfinement phase transition}

To find the conditions of the phase transition [7] we make an expansion of $\mathcal{V}^{2}(z) / \mathcal{V}^{2}\left(z_{\min }\right)$ in the Taylor series at the point $z_{\min }$ :

$$
\frac{\mathcal{V}^{2}(z)}{\mathcal{V}^{2}\left(z_{\text {min }}\right)}=1+\mathcal{V}_{1} \cdot\left(z-z_{\text {min }}\right)+\mathcal{V}_{2} \cdot\left(z-z_{\text {min }}\right)^{2}+o\left(\left(z-z_{\text {min }}\right)^{2}\right)
$$


where

$$
\begin{gathered}
\mathcal{V}_{1} \equiv \frac{2 \mathcal{V}^{\prime}\left(z_{\text {min }}\right)}{\mathcal{V}\left(z_{\text {min }}\right)}, \\
\mathcal{V}_{2} \equiv \frac{\mathcal{V}\left(z_{\text {min }}\right) \mathcal{V}^{\prime \prime}\left(z_{\text {min }}\right)+\mathcal{V}^{\prime}\left(z_{\text {min }}\right)^{2}}{\mathcal{V}^{2}\left(z_{\text {min }}\right)} .
\end{gathered}
$$

Therefore

$$
\ell=2 \int_{0}^{z_{\min }} \frac{d z}{\sqrt{\mathcal{F}(z)\left(\mathcal{V}_{1} \cdot\left(z-z_{\min }\right)+\mathcal{V}_{2} \cdot\left(z-z_{\min }\right)^{2}\right)}} .
$$

For the confinement/deconfinement phase transition the character length $\ell$ must be infinite for $z \rightarrow z_{\min }$. So we have two different cases: $\mathcal{V}^{\prime}(z)=0$ and $\mathcal{V}^{\prime}(z) \neq 0$.

1) If $\mathcal{V}^{\prime}(z) \neq 0$ we can consider the first order only:

$$
\ell=2 \int_{0}^{z_{\text {min }}} \frac{d z}{\sqrt{\mathcal{F}(z) \mathcal{V}_{1}\left(z-z_{\text {min }}\right)}} \sim \sqrt{\frac{z-z_{\text {min }}}{\mathcal{F}\left(z_{\text {min }}\right) \mathcal{V}_{1}}}
$$

so that $\ell \rightarrow 0$ as $z \rightarrow z_{\text {min }}-0$.

2) If $\mathcal{V}^{\prime}(z)=0$ we have to use the second order:

$$
\ell=2 \int_{0}^{z_{\text {min }}} \frac{d z}{\sqrt{\mathcal{F}(z) \mathcal{V}_{2}} \cdot\left(z_{\text {min }}-z\right)} \sim \frac{\log \left(z_{\text {min }}-z\right)}{\sqrt{\mathcal{F}\left(z_{\text {min }}\right) \frac{\mathcal{V}^{\prime \prime}\left(z_{\text {min }}\right)}{\mathcal{V}\left(z_{\text {min }}\right)}}},
$$

so that $\ell \rightarrow \infty$ as $z \rightarrow z_{\min }-0$. Note that $z_{\min }$ really is the minimum of function $\mathcal{V}(z)$ as $\mathcal{V}^{\prime}\left(z_{\min }\right) \equiv 0$ and

$$
\mathcal{V}(z)=\frac{M(z) \mathcal{F}(z)}{\sqrt{\mathcal{F}(z)}}>\frac{M(z) \mathcal{F}(z)}{\sqrt{\mathcal{F}(z)+z^{\prime 2}}}=\frac{M\left(z_{\text {min }}\right) \mathcal{F}\left(z_{\text {min }}\right)}{\sqrt{\mathcal{F}\left(z_{\text {min }}\right)}}=\mathcal{V}\left(z_{\text {min }}\right),
$$

thus $\mathcal{V}^{\prime \prime}\left(z_{\min }\right)>0$. So to find stationary points of $\mathcal{V}(z)$ we should solve the following equation:

$$
\mathcal{V}^{\prime}(z)=0
$$

The potential with the field of dilaton is:

$$
\mathcal{V}_{\theta}(z)=\frac{b(z) e^{\sqrt{\frac{2}{3}} \phi(z)}}{z^{2}} \sqrt{g(z)\left(z^{2-\frac{2}{v}} \sin ^{2}(\theta)+\cos ^{2}(\theta)\right)} .
$$

In our case the effective potential depends on the warp factor, the scalar field and the angle. To find stationary points of $\mathcal{V}(z)$ we solve the equation (26) for the potential (27) with arbitrary angle:

$$
\begin{aligned}
& \mathcal{D} \mathcal{W}_{\theta} \equiv c z+\frac{1}{v z} \sqrt{\frac{2}{3}} \sqrt{3 c v^{2} z^{2}\left(\frac{c z^{2}}{2}-3\right)+4 v-4}- \\
& -\frac{2}{z}+\frac{\left(1-\frac{1}{v}\right) z^{1-\frac{2}{v}} \sin ^{2}(\theta)}{\cos ^{2}(\theta)+z^{2-\frac{2}{v}} \sin ^{2}(\theta)}+\left.\frac{g^{\prime}}{2 g}\right|_{z=z D W \theta}=0 .
\end{aligned}
$$


It is possible to obtain particular cases for $\theta=0^{0}, 90^{0}[1,2]$ from the expression (28):

$$
\begin{gathered}
\mathcal{D} W_{x} \equiv c z+\frac{1}{v z} \sqrt{\frac{2}{3}} \sqrt{3 c v^{2} z^{2}\left(\frac{c z^{2}}{2}-3\right)+4 v-4}+\frac{g^{\prime}}{2 g}-\left.\frac{2}{z}\right|_{z=z_{D W_{x}}}=0, \\
\mathcal{D} \mathcal{W}_{y} \equiv c z+\frac{1}{v z} \sqrt{\frac{2}{3}} \sqrt{3 c v^{2} z^{2}\left(\frac{c z^{2}}{2}-3\right)+4 v-4+\frac{g^{\prime}}{2 g}-\left.\frac{v+1}{v z}\right|_{z=z_{D W}}=0 .} .
\end{gathered}
$$

The expression for the temperature $T\left(z_{h}, \mu, c, v\right)$ is

$$
T\left(z_{h}, \mu, c, v\right)=\frac{g^{\prime}\left(z_{h}\right)}{4 \pi}=\frac{e^{-\frac{3 c_{h}^{2}}{4}}}{2 \pi z_{h}}\left|\frac{1}{\mathfrak{5}\left(\frac{3}{4} c z_{h}^{2}\right)}+\frac{\mu^{2} c z_{h}^{2+\frac{2}{v}} e^{\frac{c z_{h}^{2}}{4}}}{4\left(1-e^{\frac{c z_{h}^{2}}{4}}\right)^{2}}\left(1-e^{\frac{c z_{h}^{2}}{4}} \frac{\mathfrak{5}\left(c z_{h}^{2}\right)}{\mathfrak{5}\left(\frac{3}{4} c z_{h}^{2}\right)}\right)\right| .
$$

Let us remind that in $[1,2]$ the thermodynamical properties of the constructed black hole background were studied and the large/small black hole phase transitions (BB-transition) were found at the temperature $T_{B B}(\mu)$. Hawking-Page phase transition takes place at $z_{h, H P}$, where the free energy equals zero. The particular value of $z_{h, H P}$ depends on $c$ and $v$ and is larger for larger negative $c$, i.e. $z_{h, H P}\left(c_{1}, v\right)<z_{h, H P}\left(c_{2}, v\right)$ for $c_{1}<c_{2}<0$. For the anisotropic background the Hawking-Page horizon is less than for the isotropic one with the same $c<0$.

At $\mu=0$ and for $T<T_{H P}(0)$ the black hole dissolves to thermodynamically stable thermal gas. If the system cools down with the non-zero chemical potential less than some critical value $\mu_{c r}$, the background undergoes the phase transition from a large to a small black hole. This is a generalization of the corresponding effect in the isotropic case [12-16]. The temperature of the large/small black hole phase transition in anisotropic case is less than in the isotropic one, i.e. $T_{B B}^{(a n i s o)}(\mu)<T_{B B}^{(i s o)}(\mu)$. The value of the critical chemical potential, up to which this phase transition exists, is bigger in the anisotropic case compared to the isotropic one, $\mu_{c r}^{(v)}>\mu_{c r}^{(i s o)}$. Also, the point $\left(\mu_{c r}^{(v)}, T_{c r}^{(v)}\right)$ for $v \rightarrow 1$ goes smoothly to $\left(\mu_{c r}^{(i s o)}, T_{c r}^{(i s o)}\right)$.

In Fig.2-4 we can see the angle dependence of the confinement/deconfinment phase transition on the Wilson loop orientation. We choose $\theta=0^{0}, 10^{\circ}, 45^{\circ}, 60^{\circ}, 90^{\circ}$. In the boundary cases the graphs coincide with the graphs for $W_{x}$ (blue solid line) and $W_{y}$ (magenta solid line) from $[1,2]$.

In our consideration we take into account the Hawking-Page phase transition (dashed pink line). For the critical angle $\theta_{c r 1}=22^{0}$ (green dashed line) the Wilson loop phase transition line intersects the Hawking-Page phase transition line at it's very end point from above. For the critical angle $\theta_{c r 2}=54^{0}$ (dashed cyan line) the top point $\mu=0$ of the confinement/deconfinement phase transition for the Hawking-Page line and the Wilson loop line is chosen to be the same. In Fig.3 we see, that in this case the Hawking-Page phase transition line coincides with the Wilson loop line along the entire length. From this angle the Wilson loop line fully determines confinement/deconfinement phase transition. This situation preserves up to the next critical angle $\theta_{c r 3}=78^{0}$ (light pink dashed line). Here the Wilson loop phase transition line intersects the Hawking-Page phase transition line at it's very end point from below. 

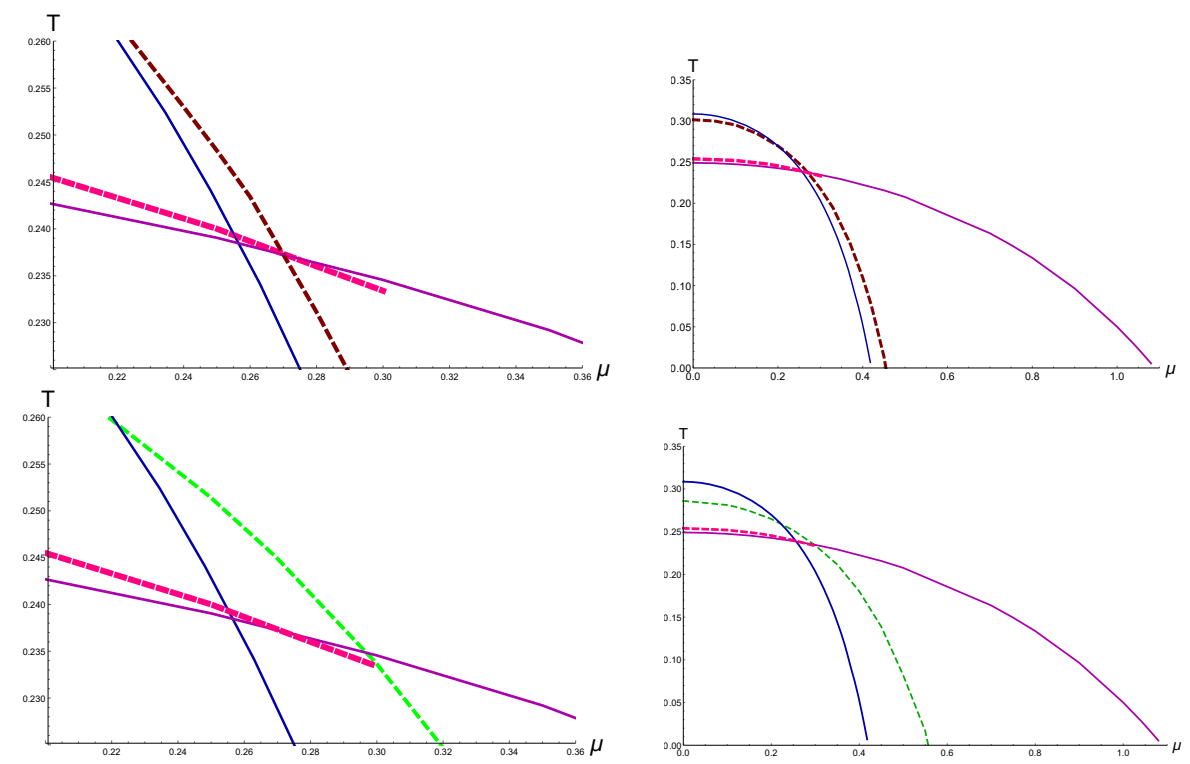

Figure 2. Phase transition diagrams for $\theta=10^{0}$ (brown dashed line, upper pictures) and $\theta_{c r 1}=22^{0}$ (green dashed line, lower pictures) in details in selected region on the left side and in general on the right side.
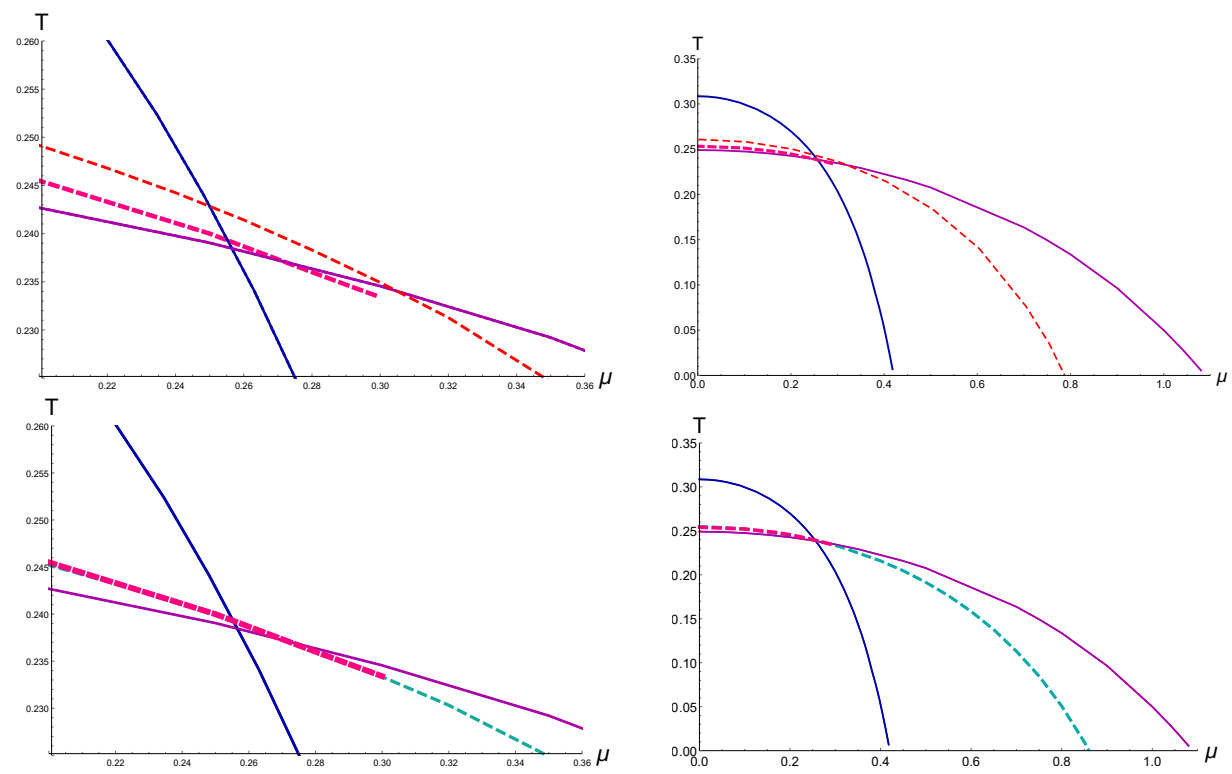

Figure 3. Phase transition diagrams for $\theta=45^{\circ}$ (red dashed line, upper pictures) and $\theta_{c r 2}=54^{0}$ (cyan dashed line, lower pictures) in details in selected region on the left side and in general on the right side. 

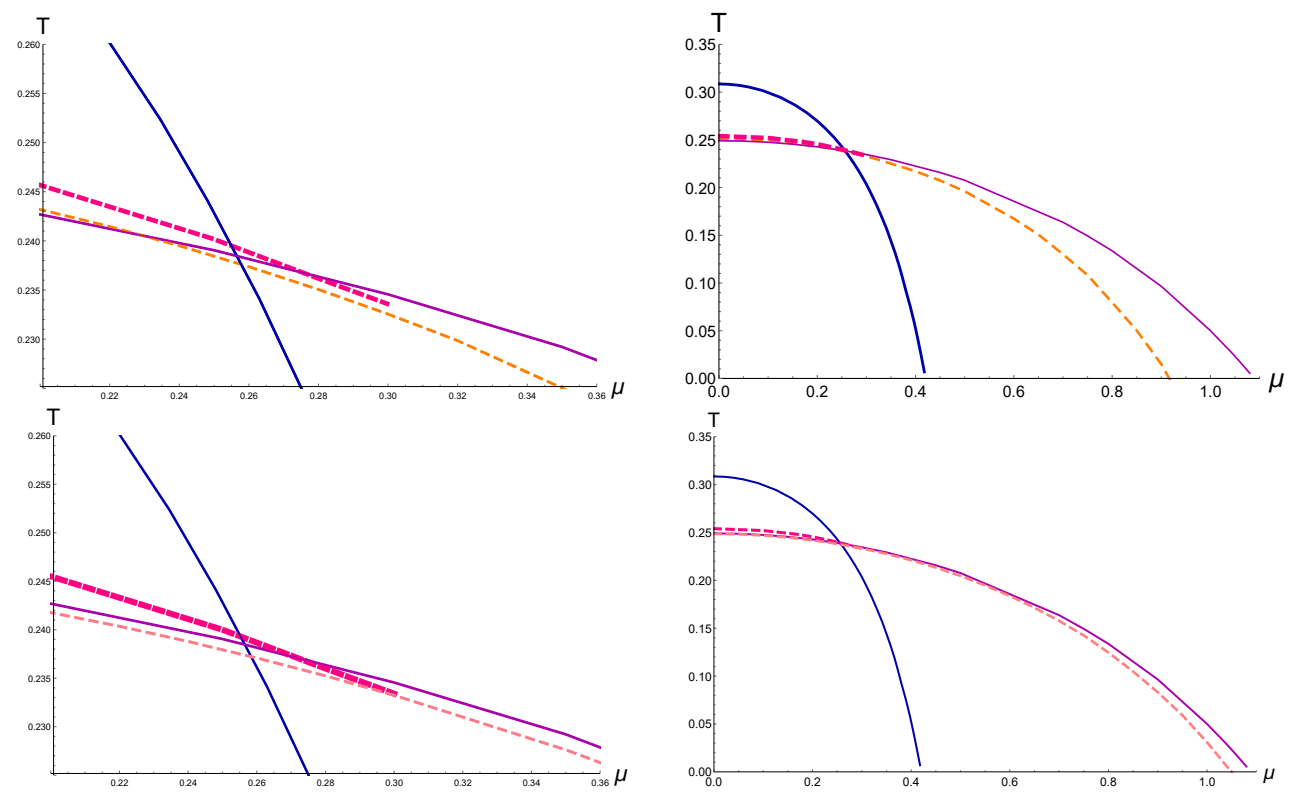

Figure 4. Phase transition diagrams for $\theta=60^{\circ}$ (orange dashed line, upper pictures) and $\theta_{c r 3}=78^{0}$ (light pink dashed line, lower pictures) in details in selected region on the left side and in general on the right side.

\section{Conclusion}

We have studied the dependence of behavior of the temporal Wilson loops on the orientation specified by arbitrary angle $\theta$ in the background (2). This result is the generalization of the two particular cases of orientation, considered in $[1,2]$, that can be associated with boundary values $\theta=0^{\circ}, 90^{\circ}$.

We demonstrated that the phase diagram depends on the orientation [7]. Taking into account the instability zones of the anisotropic background, we have found more complicated confinement/deconfinement phase diagrams for differently oriented temporal Wilson loops. For this purpose we have studied the behavior of the temporal Wilson loops in the particular 5-dimensional anisotropic background supported by dilaton and two-Maxwell field. The diagram is defined in $(\mu, T)$-plane for arbitrary angles.

In this model we have determined the critical angles $\theta_{c r 1}=22^{0}, \theta_{c r 2}=54^{0}, \theta_{c r 3}=78^{0}$. For the critical angle $\theta_{c r 1}=22^{0}$ the part of confinement/deconfinement phase transition line is determined by the Hawking-Page phase transition. For the angle $\theta_{c r 2}=54^{0}$ the top point of the Hawking-Page phase transition coincides with the top point of the confinement/deconfinement phase transition. For the $\theta_{c r 3}=78^{0}$ the whole confinement/deconfinement phase transition line is determined by the Wilson loop.

More detailed consideration for the arbitrary orientation is presented in [17]. In all likelihood these calculations are relevant in the context of the future NICA and FAIR projects.

\section{Acknowledgments}

This paper is based on a talk at the XXth International Seminar on High Energy Physics "Quarks-2018" at Valday, Russia on 27 May - 2 June 2018. Author would like to thank the 
organizers of Quarks-2018 for the support. Author would like to thank Irina Arefeva and Kristina Rannu for useful discussions.

\section{References}

[1] I. Aref'eva and K. Rannu, JHEP 05, 206 (2018)

[2] K. Rannu, talk "Holographic anisotropic background with confinement-deconfinement phase transition" on the XXth International Seminar "Quarks-2018".

[3] J. Casalderrey-Solana, H. Liu, D. Mateos, K. Rajagopal, U. Wiedemann, Cambridge University Press, (2014)

[4] I. Aref'eva, Phys. Usp. 57, 527 (2014)

[5] I. Aref'eva, talk "Holography for Heavy Ions Collisions at LHC and NICA" on the XXth International Seminar "Quarks-2018".

[6] O. DeWolfe, S. Gubser, C. Rosen and D. Teaney, Prog. Part. Nucl. Phys. 75, 86 (2014)

[7] I. Aref'eva, EPJ Web Conf. 164, 01014 (2017)

[8] I. Aref'eva, A. Golubtsova and E. Gourgoulhon, JHEP 1609, 142 (2016)

[9] J. Maldacena, Phys. Rev. Lett. 80, 4859 (1998)

[10] S. Rey, S. Theisen and J. Yee, Nucl. Phys. B 527, 171 (1998)

[11] A. Brandhuber, N. Itzhaki, J. Sonnenschein and S. Yankielowicz, Phys. Lett. B 434, 36 (1998)

[12] A. Chamblin, R. Emparan, C. Johnson and R. Myers, Phys. Rev. D 60, 064018 (1999)

[13] O. DeWolfe, S. Gubser and C. Rosen, Phys. Rev. D 83, 086005 (2011)

[14] O. DeWolfe, S. Gubser and C. Rosen, Phys. Rev. D 84, 126014 (2011)

[15] S. He, S.-Y. Wu, Y. Yang and P.-H. Yuan, JHEP 04, 093 (2013)

[16] Y. Yang and P.-H. Yuan, JHEP 1512, 161 (2015)

[17] I. Aref'eva, K. Rannu and P. Slepov, arXiv:1808.05596 [hep-th] (2018) 